\title{
Magnetically induced ferroelectricity in orthorhombic manganites: Microscopic origin and chemical trends
}

\author{
Kunihiko Yamauchi, ${ }^{1}$ Frank Freimuth, ${ }^{2}$ Stefan Blügel, ${ }^{2}$ and Silvia Picozzi ${ }^{1}, *$ \\ ${ }^{1}$ CASTI Regional Laboratory, Consiglio Nazionale delle Ricerche-Instituto Nazionale di Fisica della Materia (CNR-INFM), \\ 67100 L'Aquila, Italy \\ ${ }^{2}$ Institut für Festkörperforschung, Forschungszentrum Jülich, 52425 Jülich, Germany
}

(Received 16 February 2008; revised manuscript received 23 May 2008; published 2 July 2008)

\begin{abstract}
The microscopic origin of the magnetically driven ferroelectricity in collinear $E$-type antiferromagnetic $(\mathrm{AFM}-E)$ orthorhombic manganites is explained by means of first-principles Wannier functions. We show that the polarization is mainly determined by the asymmetric electron hopping of orbitally polarized $e_{g}$ states, implicit in the peculiar in-plane zigzag spin arrangement in the AFM- $E$ configuration. In ortho- $\mathrm{HoMnO}_{3}$, Wannier-function centers are largely displaced with respect to corresponding ionic positions, implying that the final polarization is strongly affected by a purely electronic contribution, at variance with standard ferroelectrics where the ionic displacement is dominant. However, the final value of the polarization is the result of competing effects, as shown by the opposite signs of the contributions to the polarization coming from the $\mathrm{Mn} e_{g}$ and $t_{2 g}$ states. Furthermore, a systematic analysis of the link between ferroelectricity and the spin, orbital, and lattice degrees of freedom in the manganite series has been carried out, in the aim of ascertaining chemical trends as a function of the rare-earth ion. Our results show that the Mn-O-Mn angle is the key quantity in determining the exchange coupling: upon decreasing the Mn-O-Mn angle, the first- (second-) nearest-neighbor ferromagnetic (antiferromagnetic) interaction decreases (remains constant), in turn stabilizing either the $A$-type antiferromagnetic or the AFM- $E$ spin configuration for weakly or strongly distorted manganites, respectively. The $\mathrm{Mn} e_{g}$ contribution to the polarization dramatically increases with the Mn-O-Mn angle and decreases with the "long" Mn-O bond length, whereas the Mn $t_{2 g}$ contribution decreases with the "short" $\mathrm{Mn}-\mathrm{O}$ bond length, partially canceling the former term.
\end{abstract}

DOI: 10.1103/PhysRevB.78.014403

\section{INTRODUCTION}

Multiferroics are attractive multifunctional materials where magnetism and ferroelectricity coexist; however, their coupling is often weak if measurable at all. ${ }^{1}$ In particular, orthorhombic rare-earth manganites $\mathrm{RMnO}_{3}$ represent an important class of "improper ferroelectrics,"1-3 where electric dipoles are induced by a frustrated magnetic ordering. Within this family of compounds, $\mathrm{TbMnO}_{3}$ and $\mathrm{DyMnO}_{3}$, in their noncollinear magnetic phases, have been experimentally shown to behave as multiferroics showing a weak polarization $\left(P<0.1 \mu \mathrm{C} / \mathrm{cm}^{2}\right)$ and a spin-flop ferroelectric transition. ${ }^{4}$ Moreover, it has been recently predicted that relatively strong ferroelectricity occurs in the $E$-type antiferromagnetic (AFM) phase of $R \mathrm{MnO}_{3}$ through a model study where the double-exchange interaction between Mn $d$ orbitals is proposed as a driving force for polar atomic displacements. ${ }^{5}$ In that case, the electric polarization $P$ is not related to Dzyaloshinskii-Moriya interaction, which is expected to generate much lower $P{ }^{6-8}$ Following the model study, our previously reported ab initio calculations ${ }^{9}$ have confirmed that AFM- $E \mathrm{HoMnO}_{3}$ indeed shows a high ferroelectric polarization $\left(P \sim 6 \mu \mathrm{C} / \mathrm{cm}^{2}\right)$; it was shown there that the AFM- $E$ spin ordering, which breaks the spaceinversion symmetry, is such that ferroelectric dipoles arise even without atomic displacements. ${ }^{9}$ From the experimental point of view, ferroelectricity was detected in polycrystalline ortho- $\mathrm{HoMnO}_{3},{ }^{10}$ the magnitude of polarization being however much smaller than what theoretically predicted and showing a strong dependence on the magnetic field below the ordering temperature of the Ho, suggesting their involve-
PACS number(s): 75.47.Lx, 75.80.+q, 75.50.Ee, 77.80.-e

ment in the development of $P$. The reason behind the disagreement between theory ${ }^{5,9}$ and experiments ${ }^{10}$ is still under debate.

The microscopic origin of the multiferroism in ortho- $R \mathrm{MnO}_{3}$ is tightly linked to the lattice degree of freedom: a small ionic radius of the $R$ atom directly causes the $\mathrm{GdFeO}_{3}$-like tilting of $\mathrm{MnO}_{6}$ octahedron and its interplay with the Jahn-Teller (JT) distortion leads to: (i) spin configuration determined by $\mathrm{Mn} d$ superexchange interaction $J_{i j}$, (ii) orbital ordering stabilized by JT distortion, and (iii) hopping integral in terms of double-exchange interaction. Since these aspects are all mutually combined, first we will discuss the case of AFM- $E \mathrm{HoMnO}_{3}$, and we will explain, from a microscopic quantum-mechanical point of view, the origin of the magnetically induced ferroelectric polarization in terms of the spin, orbital, and lattice degrees of freedom. The analysis based on Wannier functions represents a novel development with respect to our previous study on $\mathrm{HoMnO}_{3}{ }^{9}$ Interestingly, it shows that $\mathrm{Mn} e_{g}$ and $t_{2 g}$ states contribute in opposite ways to the polarization. Moreover, in Sec. II, we will discuss about structure, magnetism, orbital ordering, hopping integrals, and ferroelectricity-as well as the links between them-along the manganites series, with the main aim of identifying chemical trends as a function of the $R$ ion.

\section{STRUCTURAL AND COMPUTATIONAL DETAILS}

The unit cell in orthorhombic $R \mathrm{MnO}_{3}$ shows the Pnma symmetry (whereas the Pbnm setting is used in some other references, the standard Pnma orientation is adopted in this paper, i.e., we choose $b$ as the longest axis), with strong 
distortions with respect to the ideal cubic perovskite. Although the hexagonal nonperovskite phase is more stable than the orthorhombic phase in $R \mathrm{MnO}_{3}$ for $R$ smaller than $\mathrm{Tb}$, the transition to the metastable orthorhombic phase can be obtained by high-pressure synthesis. ${ }^{11-14}$ We have considered five kinds of AFM configurations: AFM- $A$, AFM- $C$, and AFM- $G$ in 20 atoms/unit cell and AFM- $E$ and AFM- $E^{*}$ in 40 atoms/unit cell. We recall that, according to the standard Wollan-Koehler notation, ${ }^{15}$ the AFM- $A$ shows ferromagnetic (FM) (AFM) intraplanar (interplanar) coupling; the AFM-C shows FM (AFM) interplanar (intraplanar) coupling; the AFM- $G$ shows AFM in both intra- and interplanar coupling. The AFM- $E$ shows in-plane FM zigzag chains antiferromagnetically coupled to the neighboring chains; the interplanar coupling is also AFM. We denote by AFM- $E^{*}$ the spin configuration showing the same in-plane spin arrangement as AFM- $E$ but with an interplanar FM coupling. Note that the AFM- $A$ spin arrangement shows the space inversion as symmetry operation at variance with the AFM- $E$ spin configuration, which shows noncentrosymmetric $P m n 2_{1}$ symmetry.

As far as the electronic structure is concerned (especially for the density of states projected on the Mn atom as well as orbital ordering, see below), it is useful to define-in addition to the "global" $X, Y, Z$ orthorhombic frame-a "local" frame, specific to each Jahn-Teller-type distorted $\mathrm{MnO}_{6}$ octahedron, obtained by choosing $x, y, z$ along the middle, short, and long Mn-O axes, respectively. In this local frame, the orbital ordered $\mathrm{Mn} e_{g}^{1}$ state, which is often expressed as $\left(3 x^{2}-r^{2}\right) /\left(3 y^{2}-r^{2}\right)$, is described as $3 z^{2}-r^{2}$ (denoted as $z^{2}$ hereafter for simplicity). The simulations were performed by using density-functional theory and the Perdew-BeckeErzenhof version of the generalized gradient approximation (GGA) to the exchange-correlation potential. ${ }^{16}$ The calculations were done with two program codes according to different purposes.

The expensive calculations for the structural optimization of the atomic structure and Berry phase for the AFM- $A$ and AFM- $E$ phases were done with "Vienna $A b$ initio Simulation Package (VASP) program code,"17 where projectoraugmented-wave potential is used. The plane-wave cutoff is set to $500 \mathrm{eV}$ in energy. We used eight special $k$ points (divided as $4 \times 3 \times 4)$ in 1/8 irreducible Brillouin zone (IBZ) for $A$-AFM phase and four special $k$ points (divided as $2 \times 3$ $\times 4)$ in $1 / 4$ IBZ for $E$-AFM phase, according to the Monkhorst-Pack scheme. The Berry phase was calculated by integrating over six $k$-point strings parallel to the $c$ axis, each string containing six $k$ points.

Calculations requiring the total-energy differences for the stability of different spin configurations) and the construction of Maximally localized Wannier functions (WFs) (Refs. 18 and 19) were done with the FLEUR code,${ }^{20}$ which is based on full-potential linearized-augmented plane-wave formalism. ${ }^{21}$ The total-energy differences for the stability of different spin-configuration have also been evaluated within FLEUR. Muffin-tin radii were set to 2.5, 2.0, and 1.5 a.u. for $R, \mathrm{Mn}$, and $\mathrm{O}$ atoms, respectively, where the wave-function cutoff was chosen as 3.8 a.u. $^{-1}$. The potential was converged with 24 special $\mathbf{k}$ points and density of states was calculated with $192 \mathbf{k}$ points within the tetrahedron method. For AFM- $E$ and AFM- $E^{*}$ phases, $12 \mathbf{k}$-points was used according to the

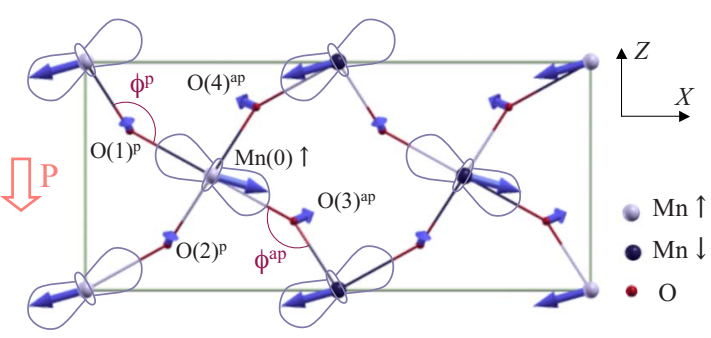

FIG. 1. (Color online) Atomic displacements in $\mathrm{FE} \mathrm{HoMnO}_{3}$, as obtained by the difference of atomic coordinates in optimized AFM- $E$ and optimized AFM- $A$ spin configurations (length of arrows in arbitrary units). In the $\mathrm{MnO}_{2}$ plane, the AFM- $E$ spin arrangement is also shown with black (up) and white (down) Mn atoms. A schematic representation of the $e_{g}$ orbital ordering and the direction of the ferroelectric polarization are also shown. The axis $X$ corresponds to $a(b)$ and $Z$ corresponds to $c(a)$ axis in the Pnma (Pbnm) setting.

doubled unit cell. The Wannier-function calculation, whose procedure was recently implemented in FLEUR code, ${ }^{22}$ was done with $512 \mathbf{k}$ points (divided as $8 \times 8 \times 8$ ). $R 5 s$ and $5 p$ states are treated as local orbitals.

The localized $R 4 f$ electrons were assumed as core electrons: "frozen core" within the VASP code and "open core" within the FLEUR code ${ }^{23}$ where the spin moment is maximized due to Hund's rule. Irrespective of these approaches, the $4 f$ states lie deep in energy (a few eV below the Fermi energy) and they are almost completely undispersed so that they do not affect other valence states.

\section{MICROSCOPIC ORIGIN OF THE FERROELECTRIC POLARIZATION IN $\mathrm{HoMnO}_{3}$}

Let us start our discussion by focusing on the case of AFM- $E \mathrm{HoMnO}_{3}$, which was previously found by some of us to show a large polarization along the in-plane $c$ axis due to noncentrosymmetric collinear spin-arrangement. ${ }^{9}$ In this work, we perform a careful analysis in terms of Wannier functions, which explains the microscopic mechanism at the basis of the macroscopic ferroelectricity. The structural parameters used in our calculations are summarized in Sec. IV, together with the parameters in other $R \mathrm{MnO}_{3}$ compounds. The magnetically induced spontaneous polarization was calculated by using: (i) the point-charge model (PCM) where each ion has been given its nominal charge (Ho: +3 , Mn:3+, O:2-), (ii) the Berry phase (BP) method with the VASP code, and (iii) the WF method with the FLEUR code. ${ }^{24}$ We recall that in the first approach, only the positions of the anions and cations are considered, whereas in the two latter ${ }^{25,26}$ quantum-mechanical treatments, the self-consistent electronic structure is fully taken into account.

To calculate the polarization within $\mathrm{BP}$, an adiabatic path from AFM- $A$ to AFM- $E$ phase is assumed, in such a way that the direction of Mn spins are progressively rotated from an in-plane FM to a zigzaglike arrangement. ${ }^{27}$ The ionic contribution from core electrons and protons are added to BP calculated for the fully occupied valence states. In Fig. 1 and Table I we show the displacements of the atoms in the noncentrosymmetric AFM- $E$ spin configuration with respect to 
TABLE I. Atomic displacements from centrosymmetric AFM- $A$ phase in ferroelectric AFM-E $\mathrm{HoMnO}_{3}(\AA) . \mathrm{O}(1)^{p}$ and $\mathrm{O}(2)^{p}$ $\left[\mathrm{O}(3)^{\text {ap }}\right.$ and $\left.\mathrm{O}(4)^{\mathrm{ap}}\right]$ are connected by $C_{2 Z}$ symmetry operation. The used notation of atoms is shown in Fig. 1.

\begin{tabular}{lrrrr}
\hline \hline & \multicolumn{1}{c}{$\Delta X$} & $\Delta Y$ & \multicolumn{1}{c}{$\Delta Z$} & $|d r|$ \\
\hline $\mathrm{Mn}(0)$ & 0.036 & 0.003 & -0.010 & 0.037 \\
$\mathrm{O}(1)^{\mathrm{p}}$ & -0.006 & 0.009 & 0.016 & 0.020 \\
$\mathrm{O}(3)^{\mathrm{ap}}$ & 0.020 & 0.010 & 0.009 & 0.024 \\
\hline \hline
\end{tabular}

the centrosymmetric AFM- $A$ structure. These quantities will be needed in the discussion reported below.

By using WFs, one can decompose the total polarization into contributions coming from each set of orbitals. For clarity, we show in Fig. 2 the AFM- $E \mathrm{HoMnO}_{3}$ insulating band structure where the relevant states are highlighted: $\operatorname{Mn} e_{g}$, $\mathrm{Mn} t_{2 g}$, and $\mathrm{O} p$ orbitals. We have projected these three groups of occupied eigenstates into real-space basis separately and "maximally localized" them to obtain the corresponding WFs. The contribution from deeper occupied valence states (such as $\mathrm{O} 2 s, R 5 s$, and $5 p$ states) is neglected in the WF approach. The total polarization of WF is the sum of the displacement of the center of each WF from the position of the corresponding ion plus PCM contribution. Other details of WF are the same as in Ref. 22.

According to the different approaches, our estimated values for the polarization in AFM- $E$ HoMnO 3 are $P_{\mathrm{BP}}=-6.14 \mu \mathrm{C} / \mathrm{cm}^{2}, \quad P_{\mathrm{WF}}=-5.61 \mu \mathrm{C} / \mathrm{cm}^{2}, \quad$ and $P_{\mathrm{PCM}}=-2.09 \mu \mathrm{C} / \mathrm{cm}^{2}$ along the $Z$ axis. The values of $P$ along the $X$ and $Y$ axes are both negligible in each approach. The large discrepancy of the values of $P_{\mathrm{WF}}\left(P_{\mathrm{BP}}\right)$ from $P_{\mathrm{PCM}}$ is notable and will be discussed in Sec. III A.

\section{A. Wannier representation of anomalous contributions}

The difference between polarization $P_{\mathrm{WF}}\left(P_{\mathrm{BP}}\right)$ and $P_{\mathrm{PCM}}$ is referred to as the anomalous contribution ${ }^{28}$ and it is analyzed by means of Wannier functions. Table II shows orbitaldecomposed polarization obtained by the displacement of the

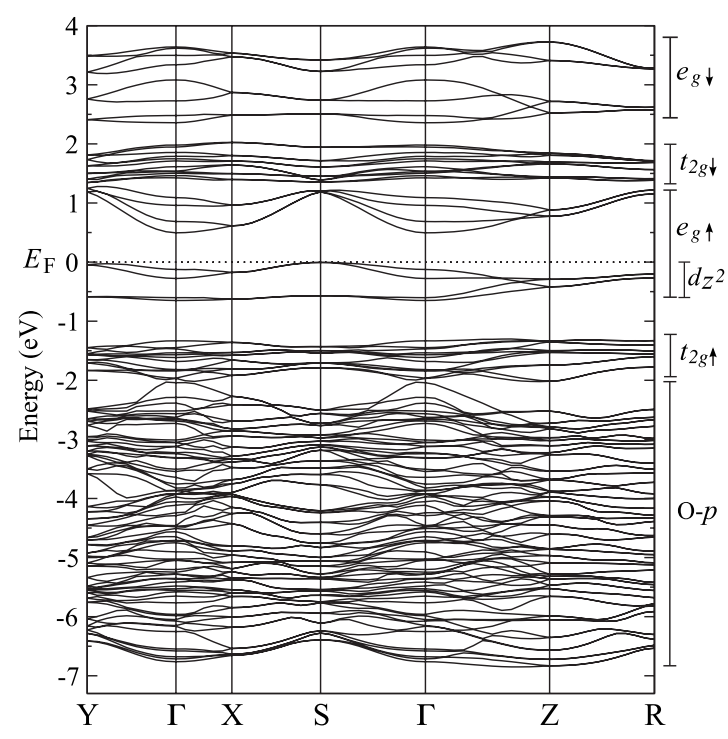

FIG. 2. Energy band structure of optimized AFM- $E \mathrm{HoMnO}_{3}$. Due to the AFM- $E$ state, only up-spin channel is plotted. $E_{F}$ denotes the Fermi energy and it is set as zero of the energy scale.

center of WF (WFC) with respect to ionic positions for each set of bands. By definition, the sum of the WFC displacements gives the difference between $P_{\mathrm{WF}}$ and $P_{\mathrm{PCM}}$, which can be regarded as the difference between the dynamical charge $Z^{*}$ and static (nominal) charge $Z$. In other words, it quantifies the effects of going from a more ionic charge distribution to more covalent bonding state. By means of the WF analysis, not only one can obtain relevant information on which orbital causes the anomalous contribution but also a real-space picture of the polar orbital states.

Let us first focus on the $e_{g}$-like $d_{z^{2}}$ and $t_{2 g}$-like $d_{y z}$ orbitals, which together with $\mathrm{O} p$ orbitals, mostly contribute to polarization (see main displacements along the polar $c$ axis in Table II). First, consider the $e_{g}$ orbital at $\mathrm{Mn}(0)$-up site surrounded by four $\mathrm{O}$ ions and four second-neighbor $\mathrm{Mn}$ ions in the $a c$ plane [Fig. 3(a)]. We recall that, within the WF formalism, the weight of each WF (centered on a specific site) on neighboring atoms reflects electron hopping from the WF-

TABLE II. WFC displacements from atomic coordinates in AFM- $E \mathrm{HoMnO}_{3}(\AA)$. The orbitals are denoted in a local frame ( $x$ : middle, $y$ : short, $z$ : long axis for Mn, $x$ : antibonding interplane, $y$ : antibonding in plane, and $z$ : bonding for $\mathrm{O})$. Only up-spin contribution is shown.

\begin{tabular}{lcrrrr}
\hline \hline \multirow{2}{*}{$\mathrm{Mn}(0)$} & $\Delta X$ & $\Delta Y$ & $\Delta Z$ & $|d r|$ \\
& $e_{g}: z^{2}$ & -0.169 & 0.044 & 0.188 & 0.257 \\
& $t_{2 g}: x y$ & 0.021 & -0.008 & -0.017 & 0.028 \\
& $t_{2 g}: y z$ & -0.049 & 0.018 & -0.146 & 0.155 \\
$\mathrm{O}(1)^{\mathrm{p}}$ & $t_{2 g}: z x$ & 0.013 & 0.007 & -0.008 & 0.016 \\
& $p_{x}$ & -0.158 & 0.071 & 0.200 & 0.265 \\
& $p_{y}$ & -0.110 & 0.048 & 0.209 & 0.241 \\
$\mathrm{O}(3)^{\mathrm{ap}}$ & $p_{z}$ & 0.004 & 0.037 & 0.108 & 0.115 \\
& $p_{x}$ & 0.020 & -0.001 & -0.026 & 0.033 \\
& $p_{y}$ & -0.025 & -0.013 & -0.038 & 0.047 \\
& $p_{z}$ & -0.171 & -0.016 & 0.048 & 0.178 \\
\hline \hline
\end{tabular}


(a) $\mathrm{Mn}-e_{g}: d_{z 2}$

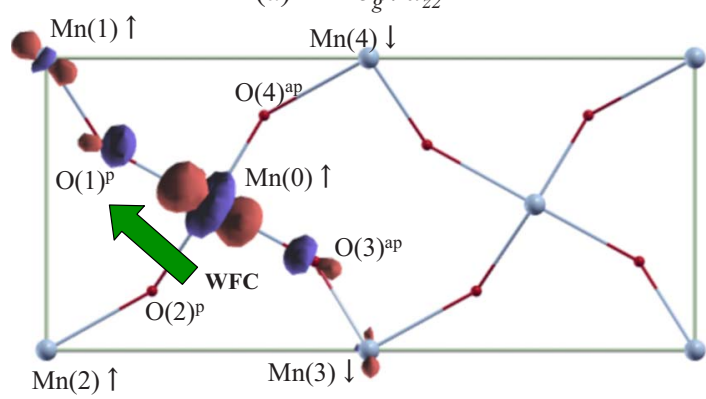

(b) $\mathrm{Mn}-t_{2 g}: d_{y z}$

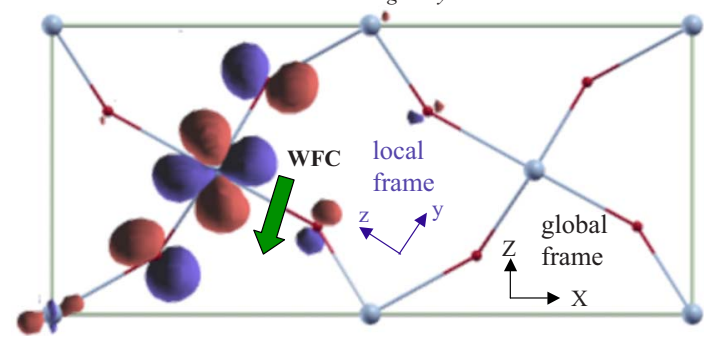

FIG. 3. (Color online) Isosurface of WFs for Mn $d$ states centered at $\mathrm{Mn}(0)$ site in AFM- $E \mathrm{HoMnO}_{3}$ : (a) $e_{g}$ states and (b) $t_{2 g}$ states. The green arrows indicate the displacement of the centers of WFs from the Mn atomic position. Here, the superscript $\mathrm{p}$ (ap) of $\mathrm{O}$ denotes oxygen ion between parallel (antiparallel) spin of Mn ions.

centered atom to states with the same spin on surrounding sites; equivalently, a large displacement of the WFC with respect to the site, on which the WF is projected, reflects a large hopping toward specific states on different sites.

In Fig. 3(a), because of the JT-derived orbital ordering, the $e_{g}: d_{z^{2}}$ orbital of $\mathrm{Mn}(0)$ ion is pointing toward the two $\mathrm{O}$ ions along long axis ( $z$ in the "local" frame). Therefore, it makes a $\sigma$ bonding with $\mathrm{O}(1)^{\mathrm{p}}$ and $\mathrm{O}(3)^{\mathrm{ap}} p_{z}$ orbitals. In addition, when considering the AFM- $E$ spin configuration, the up-spin electron of $e_{g}$ orbital of the $\operatorname{Mn}(0)$ ion can hop only onto $\mathrm{Mn}(1)$ (up spin) site via the $\mathrm{O}(1)^{\mathrm{p}}$ atom but not on the $\operatorname{Mn}(3)$ (down-spin) site. This asymmetric hopping gives the considerable deviation of the WFC from the atom position $(0.26 \AA)$ whose direction is shown as a green arrow in Fig. 3(a). Recall that the hopping integral between $\mathrm{Mn} e_{g}$ orbitals depends on both the Mn-O bond length $d$ and the Mn-O-Mn angle $\phi$, the hopping energy being therefore optimal when $\phi$ becomes larger. As a consequence, in order to increase the hopping, the $\mathrm{Mn}(0)$ ion is expected to move toward $\mathrm{O}(3)^{\text {ap }}$ to increase $\phi^{\mathrm{p}}$ between parallel $\mathrm{Mn}$ spins, i.e., along a direction that is opposite to the WFC displacement. In the same aim of increasing hopping, $\mathrm{O}(1)^{\mathrm{p}}$ moves perpendicularly to the $\mathrm{Mn}^{\uparrow}-\mathrm{Mn}^{\uparrow}$ bonding. Similarly, the $\mathrm{O}(3)^{\text {ap }}$ atom is displaced perpendicularly to the $\mathrm{Mn}^{\uparrow}-\mathrm{Mn} \downarrow$ bonding (see rough schematization of atomic displacements shown in Fig. 4). This is consistent with what shown in Fig. 1 and Table I. The ions therefore move to enhance the polarization induced by the asymmetric hopping of $e_{g}$-orbital electrons. Our proposed mechanism is quantitatively confirmed by the values of the $e_{g}$ hopping integrals from the WF centered at $\mathrm{Mn}(0)$ site to the one at $\mathrm{Mn}(1)$ site (taken from off-diagonal matrix element of the Hamiltonian, see Ref. 22): in opti-

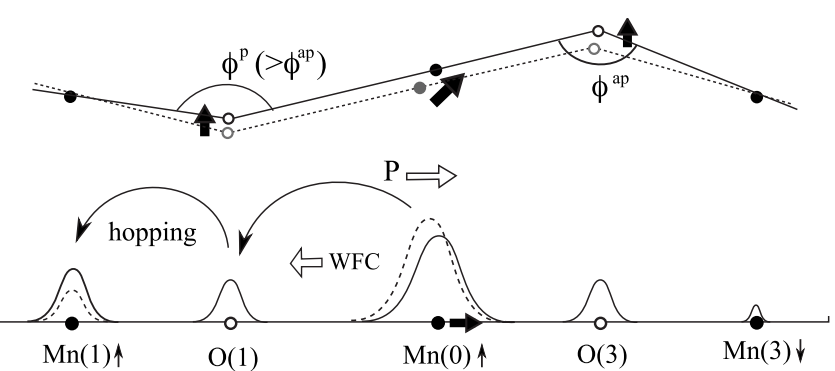

FIG. 4. Schematic representation of the mechanism which causes microscopic polarization of $\mathrm{Mn} e_{g}$ orbital due to the asymmetric electronic hopping. Bottom sketch: Hopping is allowed only from $\operatorname{Mn}(0)$ to $\operatorname{Mn}(1)$ and not to $\operatorname{Mn}(3)$ : this determines the direction of WFC (empty arrow). To increase hopping, $\mathrm{Mn}(0)$ moves "right" (see filled arrow), giving a lower weight of WF on $\mathrm{Mn}(0)$ but larger on $\mathrm{Mn}(1)$ [represented as dashed vs solid (before vs after displacement) Gaussians on the atoms]. Also shown is the resulting $\mathrm{P}$ (see arrow). Top sketch: Resulting atomic displacement (shown as filled arrows) aimed at increasing $\phi^{p}$.

mized AFM- $E$ phase $t=117 \mathrm{meV}$ and it is enhanced with respect to $t=100 \mathrm{meV}$ in optimized AFM- $A$ phase (despite the increase in the long $\mathrm{Mn}-\mathrm{O}$ bond length after ionic relaxation, which would counteract the increase in hopping given by the increased Mn-O-Mn angle). The hopping in the optimized AFM- $E$ phase is also increased with respect to $t$ $=108 \mathrm{meV}$ in optimized AFM- $A$ atomic coordinates but with the AFM- $E$ spin configuration. In parallel, the band energy (i.e., on-site energy, taken from diagonal Hamiltonian elements) related to $e_{g}$ states reduces along the adiabatic path with the ionic displacements, being minimal in the optimized AFM- $E$ phase (i.e., 8.98 vs $9.03 \mathrm{eV}$ ). Therefore, the increase in $t$ and decrease in energy in the AFM- $E$ spin configuration is mainly determined by the $\mathrm{Mn}-\mathrm{O}-\mathrm{Mn}$ angle dependence and to a less extent by the $\mathrm{Mn}-\mathrm{O}$ bond length

We further remark that the movement of the Mn ion (cf. Fig. 4) causes a current whose direction coincides with the current by the electron hopping and, therefore, it reinforces the net electronic polarization. It should be noted that the atomic displacement $(0.04 \AA)$ is very small compared to the deviation of the Wannier center $(0.26 \AA)$. We recall in fact that the atomic displacement is just a secondary effect, which occurs in order to enhance the asymmetric hopping integrals. This magnetically induced mechanism is therefore different from the conventional interpretation of polarization in standard ferroelectrics ${ }^{28}$ where the atomic displacement dominates the effect.

Next, consider the $t_{2 g}: d_{y z}$ orbital, which makes $\pi$-like bonding with surrounding oxygen $p$ orbitals. Because $d_{y z}$ orbital has isotropic symmetry in the plane, the hybridization with $p$-orbital depends only on the bond distance. In Fig. 3(b), strong hybridization of $d_{y z}$ orbital with $\mathrm{O}(2)^{\mathrm{p}} p_{z}$ and $\mathrm{O}(4)^{\text {ap }} p_{z}$ is shown. Similar to the $e_{g}$-orbital case, the electron hops mostly into $\mathrm{Mn}(2)$-up site so that the WFC is displaced in such a way [see green arrow in Fig. 3(b)]. Moreover, the atomic displacement induced by the $e_{g}$ orbitalexplained above-causes a shorter bond length between $\mathrm{Mn}(0)$ and $\mathrm{O}(3)^{\mathrm{ap}}$ ions [see weight of $\mathrm{Mn} t_{2 g} \mathrm{WF}$ on $\mathrm{O}(3)^{\mathrm{ap}}$ ]; as a consequence, the increased hybridization slightly 
(a) $\mathrm{O}^{\mathrm{p}}: p$

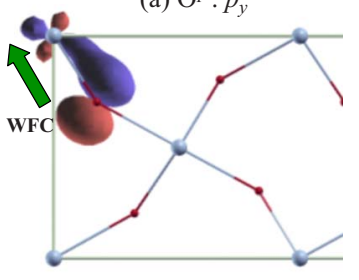

(c) $\mathrm{O}^{\mathrm{ap}}: p_{y}$

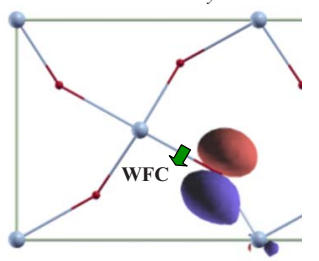

(b) $\mathrm{O}^{\mathrm{p}}: p_{z}$

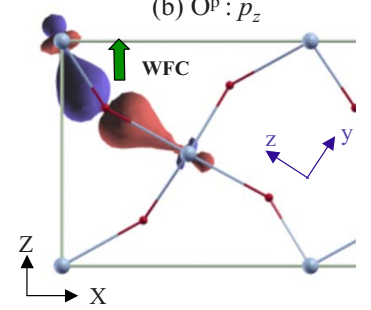

(d) $\mathrm{O}^{\mathrm{ap}}: p_{z}$

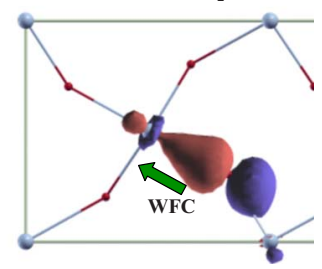

FIG. 5. (Color online) Isosurface of WFs of O $2 p$ state. Only four typical WFs in the $a c$ plane are shown. (a) $\mathrm{O}^{\mathrm{p}} p_{y}$, (b) $\mathrm{O}^{\mathrm{p}} p_{z}$, (c) $\mathrm{O}^{\text {ap }} p_{y}$, and (d) $\mathrm{O}^{\text {ap }} p_{z}$ (up-spin state). The arrows indicate the displacement of the centers of WFs from the $\mathrm{O}$ atomic position.

changes the direction of WFC displacement with respect to the $\mathrm{Mn}(0)-\mathrm{Mn}(2)$ direction. Therefore, in $\mathrm{HoMnO}_{3}$, the anomalous contributions from $\mathrm{Mn} e_{g}$ and $t_{2 g}$ orbitals almost cancel each other along the polar $c$ direction, whereas the O $p$ contribution survives. In order to complete our analysis, we therefore show in Fig. 5 the $\mathrm{O}_{\mathrm{p}}$ WF. What happens is: (i) the WFC of $\mathrm{O}^{\mathrm{p}}: p_{y}$ state (forming a $\pi$ bonding with the samespin Mn $t_{2 g}$ state) is pulled by the short-distant $\mathrm{Mn}(1)$ ion [cf. Fig. 5(a)]; (ii) the WFC of $\mathrm{O}^{\text {ap }}: p_{z}$, which makes $\sigma$ bonding with $\mathrm{Mn}(0)-e_{g}$ state is pulled by $\mathrm{Mn}(0)$ ion in the same way [cf. Fig. 5(d)]; (iii) the WFC of $\mathrm{O}^{\mathrm{p}}: p_{z}$, which forms a $\sigma$ bonding with both $\mathrm{Mn}(1)-$ and $\mathrm{Mn}(0)-e_{g}$ states is displaced toward $\mathrm{Mn}(1)$ ion but also moves to increase the Mn-O-Mn angle, with a resulting displacement roughly parallel to the $c$ axis [cf. Fig. 5(b)]; and (iv) the WFC of $\mathrm{O}^{\text {ap }}: p_{y}$, which does not form strong bonding with short-distant $\mathrm{Mn}$ ions shows a small displacement [cf. Fig. 5(c)].

\section{CHEMICAL TRENDS IN $\mathrm{RMnO}_{3}$}

From the experimental point of view, the magnetic trend of $R \mathrm{MnO}_{3}$ was found to be strongly affected by the Mn$\mathrm{O}-\mathrm{Mn}$ bond angle $\phi$ in the $\mathrm{MnO}_{2}$ plane. From $R=\mathrm{La}$ to $\mathrm{Gd}$, where $\phi=155^{\circ}-146^{\circ}$, the ground state is $A$-type AFM; upon decreasing $\phi$, there is an intermediate "lock-in incommensurate-AF state," which couples to a ferroelectric polarization at $R=\mathrm{Tb}$ and $\mathrm{Dy}$, followed by a transition to the $E$-type AFM observed from $R=$ Ho to $\mathrm{Lu}$, where $\phi=144^{\circ}$ $-140^{\circ} .29$

When $\phi$ is close enough to $180^{\circ}$, the in-plane FM coupling in AFM- $A$ phase can be explained on the basis of Kanamori-Goodenough rules ${ }^{30}$ in the framework of Mn-O "semicovalent bonding." However, when $\phi$ decreases, the overlap of $\mathrm{Mn}$ and $\mathrm{O}$ orbitals becomes smaller and Kanamori-Goodenough rules are not sufficient to explain the complex phase diagram of manganites. Instead, the nextnearest-neighbor antiferromagnetic superexchange becomes relatively dominant and the $E$-type AFM state becomes stable. ${ }^{29}$ In Sec. IV A, we will address quantitatively these general arguments and discuss how magnetic interactions are affected by $\phi$.

\section{A. Structural and magnetic properties}

\section{Atomic optimization in the AFM-A phase}

The atomic structure of $R \mathrm{MnO}_{3}$ compounds has been investigated by neutron powder diffraction ${ }^{14}$ (for $R=\mathrm{Pr}, \mathrm{Nd}$, Dy, Tb, Ho, Er, and Y), single-crystal x-ray diffractometry ${ }^{13}$ (for $R=\mathrm{Nd}$, Sm, Eu, and $\mathrm{Gd}$ ), and synchrotron $\mathrm{X}$-ray powderdiffraction measurement ${ }^{12}$ (for $R=\mathrm{Ho}, \mathrm{Er}, \mathrm{Tm}, \mathrm{Yb}$, and $\mathrm{Lu}$ ).

First, in order to discuss the magnetic stability in a tiny range of energy, we have used the experimental lattice parameters, where the volume of the unit cell linearly decreases in the series according to the Lanthanide contraction upon increasing the atomic number of $R$ atom; we have optimized the internal structural parameters imposing AFM- $A$ spin configuration on Mn $d$ electrons. The reason why we used the AFM- $A$ configuration is based on the fact that most of these compounds (where $R=\mathrm{La}$ to $\mathrm{Gd}$ ) show as ground state the AFM- $A$ spin configuration. Second, to discuss ferroelectric properties, the atomic structure was optimized with AFM- $E$ spin configuration, which, as discussed for $\mathrm{HoMnO}_{3}$, breaks the inversion symmetry of the system and leads to a magnetically induced polarization. The results of this second part will be discussed in Sec. IV B.

The optimized structural parameters in AFM- $A$ spin configuration, reported in Table III and Fig. 6, give a rather regular trend in the series. As for the $\mathrm{Mn}-\mathrm{O}$ bond length, $m$ and $s$ look rather constant over the series, whereas $l$ shows a broad maximum at $\mathrm{Gd}$. Although the calculated interplane length of $m$ shows good agreement with the experimental parameter, the in-plane length of $l$ and $s$ shows some deviation $(<10 \%)$ with respect to experiments so that the JT distortion is underestimated in this calculation. It can be argued that this problem derives from the lack of an exact treatment of the exchange-correlation potential (and/or many-body effects) at the local-density approximation (LDA)/GGA level: in our simulations, the rather correlated Mn $d$ electrons are likely to be described as more delocalized than in real manganites. ${ }^{31}$ In order to see if the effective correlation potential on Mn $d$ electrons can improve the discrepancies with experiments, we have checked the validity of LDA $+U / \mathrm{GGA}+U$ treatments, where the parameters were set as $U=8 \mathrm{eV}$ and $J=0.11 U$ (as in Ref. 31). As shown in Fig. 6(a), for rather undistorted manganites, both treatments (especially the $\mathrm{LDA}+U$ treatment) improve the calculated $\mathrm{Mn}-\mathrm{O}$ distances, which get closer to the experimental values for $\mathrm{LaMnO}_{3}$. For example, the length of JT-distortion vector $\mathbf{Q} \equiv[\sqrt{l-s}, \sqrt{2 / 3}(2 m-l-s)]$ is $Q=0.31 \AA$ within GGA, $0.32 \AA$ within $\mathrm{GGA}+U$ and $0.36 \AA$ (comparable with the value $0.4 \AA$ obtained in Ref. 31) within $\mathrm{LDA}+U$, whereas the experimental value is $0.41 \AA .{ }^{14}$ This reflects the enhancement of the on-site electron-electron interaction between $\mathrm{Mn} d$ electrons induced by the effective potential so that the $\mathrm{OO}$ is stabilized. However, as we move to more distorted manganites, the agreement with experiments is 
TABLE III. Structural parameters optimized with AFM-A configuration in Pnma unit cell where the origin is fixed at the position on Mn atom. The lattice parameter used for calculations are obtained from Ref. 14 for La, Pr, Nd, Tb, Dy, and Ho, from Ref. 12 for Er, Tm, Yb, and $\mathrm{Lu}$, and from Ref. 13 for $\mathrm{Sm}, \mathrm{Eu}$, and $\mathrm{Gd}$.

\begin{tabular}{|c|c|c|c|c|c|c|c|c|c|c|c|}
\hline & $\mathrm{La}$ & $\operatorname{Pr}$ & $\mathrm{Nd}$ & $\mathrm{Sm}$ & Gd & $\mathrm{Tb}$ & Dy & Ho & $\mathrm{Er}$ & $\mathrm{Tm}$ & $\mathrm{Lu}$ \\
\hline$a(\AA)$ & 5.7473 & 5.8129 & 5.8317 & 5.8620 & 5.8660 & 5.8384 & 5.8337 & 5.8354 & 5.8223 & 5.8085 & 5.7868 \\
\hline$b(\AA)$ & 7.6929 & 7.5856 & 7.5546 & 7.4770 & 7.4310 & 7.4025 & 7.3778 & 7.3606 & 7.3357 & 7.3175 & 7.2959 \\
\hline$c(\AA)$ & 5.5367 & 5.4491 & 5.4170 & 5.3620 & 5.3180 & 5.2931 & 5.2785 & 5.2572 & 5.2395 & 5.2277 & 5.1972 \\
\hline \multicolumn{12}{|c|}{$R 4 c\left(x \frac{1}{4} z\right)$} \\
\hline$x$ & 0.0508 & 0.0654 & 0.0714 & 0.0785 & 0.0829 & 0.0836 & 0.0846 & 0.0856 & 0.0864 & 0.0867 & 0.0870 \\
\hline$z$ & 0.4907 & 0.4866 & 0.4849 & 0.4827 & 0.4816 & 0.4808 & 0.4806 & 0.4805 & 0.4798 & 0.4799 & 0.4800 \\
\hline \multicolumn{12}{|c|}{$\operatorname{Mn} 4 a\left(\begin{array}{lll}0 & 0 & 0\end{array}\right)$} \\
\hline \multicolumn{12}{|c|}{$\mathrm{O} 14 c\left(x \frac{1}{4} z\right)$} \\
\hline$x$ & 0.4856 & 0.4820 & 0.4798 & 0.4745 & 0.4683 & 0.4665 & 0.4643 & 0.4617 & 0.4600 & 0.4574 & 0.4536 \\
\hline$z$ & 0.5779 & 0.5838 & 0.5888 & 0.5979 & 0.6066 & 0.6102 & 0.6133 & 0.6162 & 0.6190 & 0.6223 & 0.6267 \\
\hline \multicolumn{12}{|c|}{$\mathrm{O} 28 d\left(\begin{array}{lll}x & y & z\end{array}\right)$} \\
\hline$x$ & 0.3014 & 0.3079 & 0.3124 & 0.3181 & 0.3224 & 0.3223 & 0.3235 & 0.3250 & 0.3250 & 0.3258 & 0.3272 \\
\hline$y$ & 0.0404 & 0.0429 & 0.0452 & 0.0485 & 0.0515 & 0.0530 & 0.0541 & 0.0550 & 0.0561 & 0.0573 & 0.0591 \\
\hline$z$ & 0.2215 & 0.2155 & 0.2117 & 0.2071 & 0.2033 & 0.2020 & 0.2006 & 0.1988 & 0.1981 & 0.1972 & 0.1952 \\
\hline
\end{tabular}

definitely worse within GGA $+U$ than within bare GGA.

As for the Mn-O-Mn bond angle, it decreases almost linearly with the $R$ ionic radius. The interplane angle becomes larger than in-plane angle for small values of the radius or $R$ ion. Moreover, the difference between the two Mn-O distance $(l$ and $s$ ) becomes rather constant at $R=\mathrm{Gd}$. This implies that the JT distortion is rather "saturated" compared with the $\mathrm{GdFeO}_{3}$-like tilting in a $\mathrm{Mn}-\mathrm{O}_{6}$ octahedron. We note that the effective potential $U$ (both within $\mathrm{LDA}+U$ for $\mathrm{LaMnO}_{3}$ and for GGA $+U$ in all the series) worsens the agreement of the Mn-O-Mn angle with respect to the experimental value. Since the Mn-O-Mn angle is by far more important than the $\mathrm{Mn}-\mathrm{O}$ distance to discuss magnetic and ferroelectric properties (see below), we will adopt, in what follows, the structure obtained within bare GGA. In this regard, we also point out that the other reason for using the GGA potential is related to the magnetic stability, which will be explained in Sec. IV A 2.

\section{Magnetic stability}

Using the optimized atomic structure obtained by imposing the AFM- $A$ spin configuration, the total energy of AFM- $A$, AFM- $C$, AFM- $G$, AFM- $E$, and AFM- $E^{*}$ was calculated [Fig. 7(a)]. This result shows a very clear trend, which gives the transition from AFM- $A$ phase to AFM- $E$ phase at $\mathrm{SmMnO}_{3}$. Although the optimization imposing AFM- $A$ spin ordering enhances the stability of $\mathrm{AFM}-A$, the results show that the AFM- $E$ phase is most stable at $R=\mathrm{Gd}$ at variance with experimental results reporting that the AFM- $A$ phase is the spin ground state even in $\mathrm{GdMnO}_{3}$. The disagreement with experiments may again come from neglecting manybody effects within LDA/GGA. We remark that the use of an LDA/GGA $+U$ effective potential ${ }^{32}$ is not likely to improve the agreement with experiments (in addition to the fact that the value of $U$ is unknown for most of the compounds). For example, in our previous calculations, ${ }^{33}$ small values of $U$ $(\sim 2.5 \mathrm{eV})$ were found to stabilize AFM- $A$ phase with respect to AFM- $E$ in distorted manganites such as $\mathrm{HoMnO}_{3}$ at variance with experiments, which show the AFM- $E$ as ground state. ${ }^{10,34}$ In order to avoid any additional parameters and to discuss the trend of the manganites series without further bias, we do not employ the LDA/GGA $+U$ approach and focus on qualitative prediction of chemical trends (as a function of the rare-earth ion), which are expected to be well reproduced within a bare GGA approach.

Using Heisenberg Hamiltonian with normalized spin moment,

$$
\mathcal{H}=\sum_{\langle i, j\rangle} J_{i j} \vec{s}_{i} \cdot \vec{s}_{j} /\left|\vec{s}_{i}\right|\left|\vec{s}_{j}\right| .
$$

we estimated the superexchange interaction energies $J_{i j}$. From the following six equations, the difference of total energy between each AFM phase and the reference FM phase is calculated. Then using a least square mean method, we obtained four parameters for $J_{i j}$ : the first-nearest-neighbor $J_{\|}^{n n}$ and second-nearest-neighbor couplings along the $a$ axis $J_{\|}^{n n n}$ in the $a c$ plane, as well as the first- and second-nearestneighbor couplings in the out of plane $J_{\perp}^{1}$ and $J_{\perp}^{2}$. With these considered five AFM configurations, the contribution from second-nearest-neighbor coupling along the $c$ axis is not taken into account and, therefore, cannot be determined.

$$
\begin{gathered}
\mathrm{FM}: E=4 J_{\|}^{\mathrm{nn}}+2 J_{\|}^{\mathrm{nnn}}+2 J_{\perp}^{1}+8 J_{\perp}^{2}, \\
\text { AFM- } A: E=4 J_{\|}^{\mathrm{nn}}+2 J_{\|}^{\mathrm{nnn}}-2 J_{\perp}^{1}-8 J_{\perp}^{2}, \\
\text { AFM- } C: E=-4 J_{\|}^{\mathrm{nn}}+2 J_{\|}^{\mathrm{nnn}}+2 J_{\perp}^{1}-8 J_{\perp}^{2}, \\
\text { AFM- } G: E=-4 J_{\|}^{\mathrm{nn}}+2 J_{\|}^{\mathrm{nnn}}-2 J_{\perp}^{1}+8 J_{\perp}^{2}, \\
\mathrm{AFM}-E: E=-2 J_{\|}^{\mathrm{nnn}}-2 J_{\perp}^{1},
\end{gathered}
$$




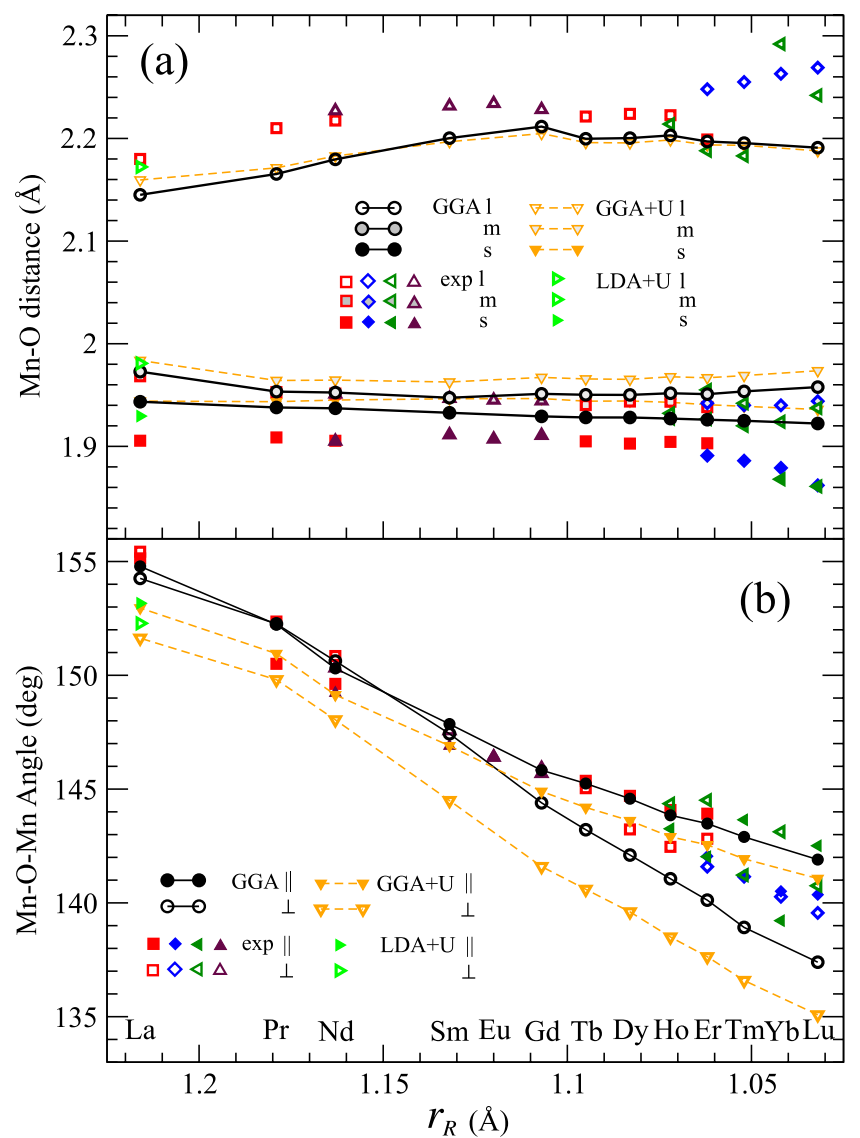

FIG. 6. (Color online) (a) Mn-O bond distance of $R \mathrm{MnO}_{3}$ as a function of $r_{R}$ (radius of rare-earth ion $R^{3+}$ ). Our results are shown as round symbols: open and filled circles show long and small Mn-O distances in $a c$ plane, respectively, and the half-filled symbols show a middle $\mathrm{Mn}-\mathrm{O}$ distance along the $b$ axis. The lines are plotted for an eye guide. (b) Mn-O-Mn angles of $R \mathrm{MnO}_{3}$ as a function of the radius of rare-earth atom. The open symbol shows interplane $\mathrm{Mn}-\mathrm{O}-\mathrm{Mn}$ angles (with apical $\mathrm{O}$ along the $b$ axis), whereas the closed symbol shows in the $a c$ plane Mn-O-Mn angles. In both panels, results obtained using GGA $+U$ (with dashed lines and down-triangles) and $\mathrm{LDA}+U$ (with right-triangles only at $\mathrm{LaMnO}_{3}$ ) are also shown. The experimental data are also shown for comparison: square from Ref. 14, diamond from Ref. 12, left triangle from Ref. 11, and upper triangle from Ref. 13.

$$
\operatorname{AFM}-E^{*}: E=-2 J_{\|}^{\mathrm{nnn}}+2 J_{\perp}^{1} .
$$

As shown in Fig. 7(b), consistently with KanamoriGoodenough rules, ${ }^{30}$ ferromagnetic $J_{\|}^{n n}$ decreases with $R$. $J_{\|}^{n n}$ represents the sum of two competing interactions (FM coupling due to $e_{g}$ orbitals and AFM coupling due to $t_{2 g}$ orbitals); the global FM behavior shows that the former dominates. However, when $\phi$ decreases, the orbital overlap between $\mathrm{Mn} e_{g}$ orbitals making $\sigma$ bonding with $\mathrm{O} p$ orbital is strongly reduced and this reduces the FM character of $J_{\|}^{n n}$, which shows a drastic reduction for small $\phi$ values. The in-plane antiferromagnetic $J_{\|}^{n n n}$ stays rather constant at variance with what proposed in the model study by Kimura et $a l .,{ }^{29}$ where $J_{\|}^{n n n}$ increases with $\phi$. The interplane coupling is expected to be AFM due to its mainly $t_{2 g}$ character. The fact

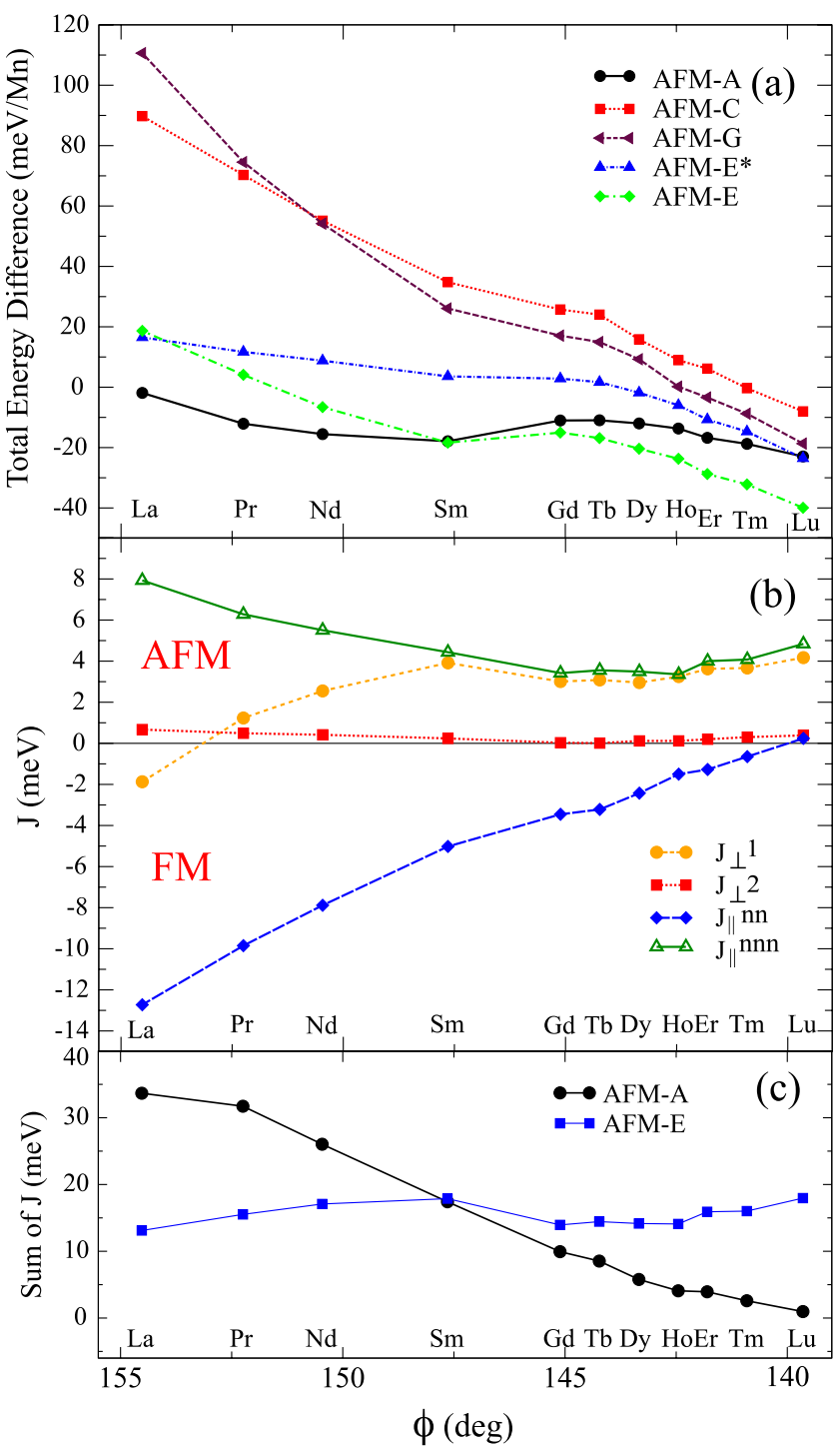

FIG. 7. (Color online) (a) Total energy of AFM phases with respect to FM phase. (b) Superexchange energy $\left(J_{i j}\right)$ : The first nearest-neighbor in plane $J_{\|}^{\mathrm{nn}}$, next-nearest-neighbor in plane $J_{\|}^{\mathrm{nnn}}$, first nearest-neighbor inter plane $J_{\perp}^{1}$, and next-nearest-neighbor interplane $J_{\perp}^{2}$. (c) Sum of $J_{i j}$ for the AFM- $A$ and AFM- $E$ phases.

that it is estimated to be weakly FM $(<2 \mathrm{meV})$ in $\mathrm{LaMnO}_{3}$ casts some doubts about the validity of a bare GGA treatment for $\mathrm{LaMnO}_{3}$ (where the AFM-A spin state is found basically degenerate with the FM spin configuration, obviously at variance with experiments). However, the correct spin ground state is reproduced for most of the $R \mathrm{MnO}_{3}$ [with exceptions of (i) the above mentioned Gd and (ii) $\mathrm{Tb}$ and Dy where we did not attempt to simulate the noncollinear spiral arrangements due to further complexity in the simulations]. ${ }^{29}$

Figure 7(c) shows the sum of the exchange constants [i.e., Eqs. (3) and (6) with sign changed]: ${ }^{35}$ within the mean-field approximation, this energy is supposed to be proportional to the ordering Néel temperature $T_{N}$. Indeed, the trend is in good agreement with experimental results, showing a steeply decreasing $T_{N}$ with AFM- $A$ in the first half of the series and a rather constant $T_{N}$ with AFM-E in the second half. As a summary of this section, an increase in $\phi$ strongly reduces 


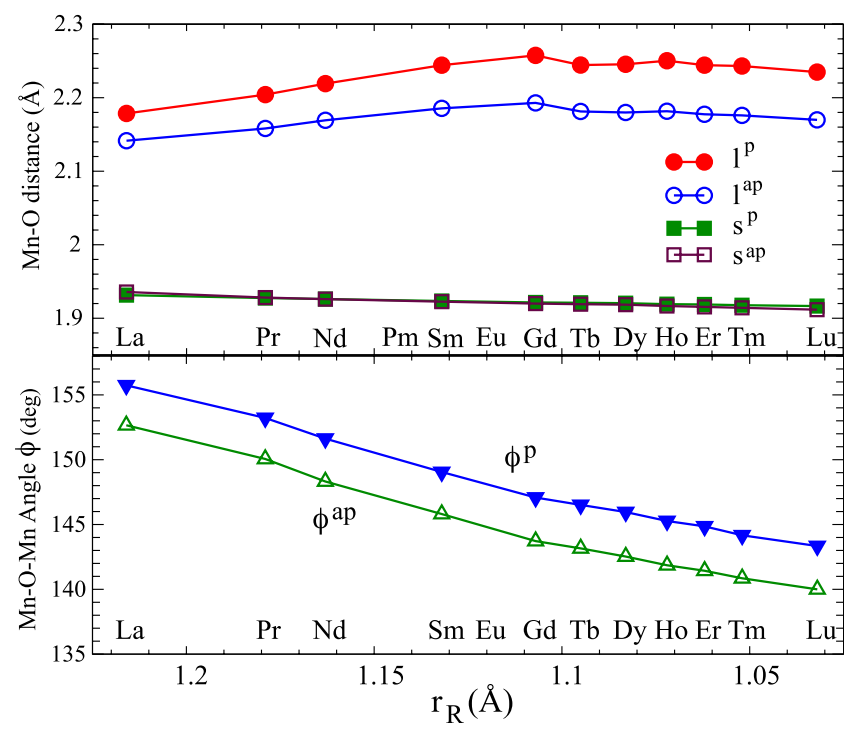

FIG. 8. (Color online) The Mn-O bond length and Mn-O-Mn bond angle as a function of $r_{R}$ in $R \mathrm{MnO}_{3}$ structure optimized in AFM- $E$ phase. Here, the superscript p (ap) denotes parallel (antiparallel) spin of Mn ions.

$e_{g}$-derived $J_{\|}^{n n}$ but does not change drastically the $J_{\|}^{\mathrm{nnn}}$ exchange constant so that a spin transition from the AFM-A phase to the AFM-E phase occurs.

\section{B. Multiferroic AFM-E spin configuration}

\section{Structural properties}

In order to discuss the chemical trends of ferroelectric properties, we optimized the internal atomic positions in the $R \mathrm{MnO}_{3}$ systems by artificially imposing the AFM- $E$ spin configuration for all $R \mathrm{MnO}_{3}$ (irrespective of whether or not the AFM- $E$ is the spin ground state). The resulting symmetry (lowered by the spin configuration with respect to the AFM- $A$ spin arrangements) allows two significantly different values of $l$ and $\phi$ between parallel and antiparallel Mn spins, whereas the optimized $s$ does not change (cf. Fig. 8). This is consistent with our mechanism proposed for $\mathrm{HoMnO}_{3}$ in Sec. IV A: since the long Mn-O bond length is mostly affected by the ferroelectric AFM- $E$ spin configuration (cf. Fig. 8 ) and it is the one along which the Mn $d_{z^{2}}$ orbital is pointing, it is likely that this latter orbital plays a key role in the resulting ferroelectricity. We also note that, along the series, the difference between $\phi^{p}$ and $\phi^{a p}$ stays rather constant; since, according to Ref. 5, this difference is at the basis of the polar atomic displacements, this constant behavior will be relevant in the discussion of polarization trends vs $R$ (see below).

\section{Electronic states and orbital ordering}

In order to better understand the trends of the ferroelectric properties as a function of $R$, reported in the following section, let us briefly discuss the electronic structure of the compounds in the AFM- $E$ spin configuration. As far as the magnetic moment in the Mn muffin-tin sphere is concerned, it is basically constant and equal to $\sim 3.3 \mu_{B}$ for all $R$. However,

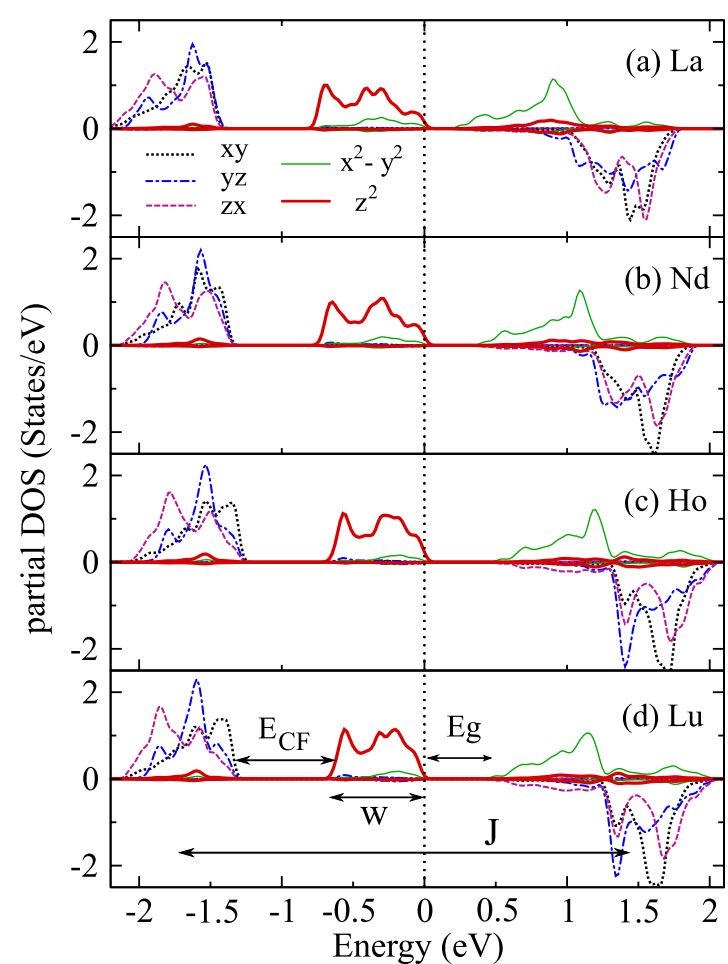

FIG. 9. (Color online) The orbital-decomposed partial DOS of Mn $3 d$ components for $R \mathrm{MnO}_{3}$, with $R$ as (a) La, (b) Nd, (c) Ho, and (d) Lu. The Arrows indicate energy gap $\left(E_{g}\right)$, crystal-field splitting $\left(E_{\mathrm{CF}}\right), e_{g}$ bandwidth $(w)$, and on-site exchange interaction energy $(J)$. The crystal structure was optimized imposing the AFM- $E$ spin configuration. The local frame in the $\mathrm{MnO}_{6}$ octahedron is chosen as $x$ : middle (interplane), $y$ : short axis, and $z$ : long axis.

some differences arise when looking at the partial density of states for Mn $3 d$ state along the series (projected in the "local" octahedron frame to highlight orbital-ordering), as reported in Fig. 9. It is clearly evident that the $d_{z^{2}}$ and $d_{x^{2}-y^{2}}$ states are fully orbitally polarized. More quantitatively, the coefficient of the $d_{z^{2}}$ orbital, obtained by diagonalization of density matrix for five $d$ orbitals, is 0.98 at $R=\mathrm{La}$ and 0.96 at $R=\mathrm{Lu}$. Being this coefficient close to one, it implies that the orbital ordering is already "saturated" at $\mathrm{LaMnO}_{3}$.

A progressive distortion of the structure (from La to $\mathrm{Lu}$ ) is shown to increase the energy gap $E_{g}$ and to decrease the width of the $e_{g}$ band $(w)$. Within a tight-binding framework, $w$ is proportional to the hopping integral $t$. Indeed, we estimated the hopping integral for selected compounds along the series and found that $t$ increases with the ionic radius of the $R$ ion (see Table IV).

Here, we would like to point out that the DOS does not change significantly before and after FE atomic displacements at variance with standard $\mathrm{FE}$ (such as $\mathrm{BaTiO}_{3}$ ), where FE atomic displacements are accompanied by a rehybridiza-

TABLE IV. Hopping integral between Mn $e_{g}$ WFs with same spin state in optimized AFM-E $R \mathrm{MnO}_{3}(\mathrm{meV})$.

\begin{tabular}{cccccc}
\hline \hline & $R=\mathrm{La}$ & $\mathrm{Nd}$ & $\mathrm{Sm}$ & $\mathrm{Ho}$ & $\mathrm{Lu}$ \\
\hline$t$ & 128 & 128 & 124 & 117 & 112 \\
\hline \hline
\end{tabular}




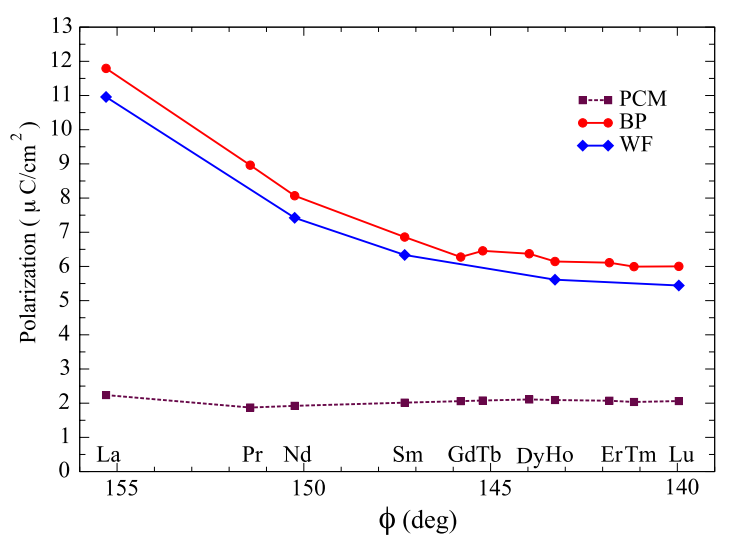

FIG. 10. (Color online) Ferroelectric polarization calculated by BP method, WF, and PCM as a function of $\phi$ in AFM- $E R \mathrm{MnO}_{3}$ (see text for details). The in-plane Mn-O-Mn bond angle $\phi$ is averaged between $\phi_{p}$ and $\phi_{\text {ap }}$.

tion of filled $\mathrm{O} p$ and empty cation $d$ states ${ }^{36}$ Here, we recall that the Mn $d$ state is well localized and the character of the $\mathrm{Mn}-\mathrm{O}$ bond is rather ionic in nature so it is not expected to undergo drastic changes upon development of polarization as far as the bonding properties are concerned.

\section{Ferroelectricity and link to lattice, spin, and orbital degrees of freedom}

Similarly to the case of $\mathrm{HoMnO}_{3}$, we have calculated the polarization according to the PCM, BP, and WF approaches. As shown in Fig. 10, the polarization calculated by BP and WF are consistent within less than 10\%: this difference (in addition to possible numerical uncertainties deriving from the use of a different basis set and potential treatments in VASP and FLEUR codes for BP and WF approaches, respectively) comes from $\mathrm{O} 2 s, R 5 s$, and $R 5 p$ contributions and shows that these latter contributions, as expected, are small compared to $\mathrm{Mn} d$ and $\mathrm{O} p$ contributions.

What is truly remarkable is that the polarization (both from $\mathrm{BP}$ and WF) shows a rapid increase upon increasing $\phi$ $\left(P_{\mathrm{BP}}\right.$ approaches $12 \mu \mathrm{C} / \mathrm{cm}^{2}$ in an hypothetical AFM-E $\mathrm{LaMnO}_{3}$ ), whereas $P_{\mathrm{PCM}}$ is to a large extent constant. This latter trend is consistent with the constant behavior of $\phi^{p}$ $-\phi^{a p}$ shown in Fig. 8 and with what previously discussed in Refs. 5 and 9. Moreover, the difference between $\mathrm{P}_{\mathrm{BP}} / \mathrm{P}_{\mathrm{WF}}$ and $\mathrm{P}_{\mathrm{PCM}}$ (which represents a purely electronic contribution) is much bigger than the contribution coming from atomic displacements in the entire series.

Our results further suggest that orbital ordering is necessary for the rising of the polarization; however, being constant along the series, is not responsible for the trend of FE polarization, which, on the other hand, shows dramatic changes as a function of $R$. Consistently with what previously discussed for $\mathrm{HoMnO}_{3}$ where $t$ is suggested to play an important role, the dramatic increase in the asymmetric $e_{g}$ hopping is responsible for enhancing $P$ in less distorted manganites (cf. Table $\mathrm{V}$ where we report the different contribution of $\mathrm{Mn} e_{g}, \mathrm{Mn} t_{2 g}$, and $\mathrm{O} p$ to the total $\left.P_{\mathrm{WF}}\right)$. We show that, for every $R$, the $\mathrm{Mn} e_{g}$ and $\mathrm{O} p$ contributions show an opposite sign with respect to the Mn $t_{2 g}$ term with regular
TABLE V. Polarization $\left(\mu \mathrm{C} / \mathrm{cm}^{2}\right)$ induced by the displacement of WFC from the ionic position in AFM- $E R \mathrm{MnO}_{3}$. Only up-spin contribution is reported for each WF: $\mathrm{Mn} e_{g}, \mathrm{Mn} t_{2 g}$, and $\mathrm{O} p$. In the two final lines, we show the sum of the contributions (for spin-up only) and the total (for spin-up and spin-down).

\begin{tabular}{lrrrrr}
\hline \hline$P$ & $R=\mathrm{La}$ & \multicolumn{1}{c}{$\mathrm{Nd}$} & \multicolumn{1}{c}{$\mathrm{Sm}$} & \multicolumn{1}{c}{$\mathrm{Ho}$} & \multicolumn{1}{c}{$\mathrm{Lu}$} \\
\hline $\mathrm{Mn} e_{g}$ & -4.76 & -3.48 & -3.01 & -2.67 & -2.61 \\
$\mathrm{Mn} t_{2 g}$ & 1.36 & 1.73 & 2.02 & 2.42 & 2.47 \\
$\mathrm{O} p$ & -0.96 & -1.00 & -1.17 & -1.51 & -1.55 \\
Total (up) & -4.36 & -2.75 & -2.16 & -1.76 & -1.69 \\
Total (up+down) & -8.72 & -5.50 & -4.32 & -3.52 & -3.38 \\
\hline
\end{tabular}

trends along the series. Indeed, remembering that the hopping integral $t$ depends on the bond length and bond angle, one expects smaller $l$ and larger $\phi$ to enhance $t$ between $e_{g}$ orbitals, whereas smaller $s$ to enhance $t$ between $t_{2 g}$ orbitals; this is confirmed by looking carefully at Table V and Fig. 8 and their implications for the values of P (cf. Fig. 10).

\section{CONCLUSIONS}

Ferroelectricity, recently proposed for the collinear AFM- $E$ magnetic phase in orthorhombic $\mathrm{HoMnO}_{3}$, is here explained microscopically from first principles via a careful Wannier-function analysis. We show that the asymmetric electron hopping of orbitally polarized $\mathrm{Mn} e_{g}$ states is the key ingredient for the rising of polarization. At variance with proper ferroelectrics, in $\mathrm{HoMnO}_{3}$ the purely electronic contribution (due to Wannier-function centers, which are largely displaced with respect to ions) dominates the polarization with respect to the contribution to $P$ coming from ionic movements. However, the net polarization along the $c$ axis is the result of a delicate balance of different contributions, such as, for example, the opposite signs of the Mn $e_{g}$ and $t_{2 g}$ contributions to $P$.

In addition, extensive $a b$ initio calculations have been performed for the $R \mathrm{MnO}_{3}$ systems, focusing on the link between ferroelectricity and the spin, orbital, and lattice degrees of freedom in the aim of identifying chemical trends along the series. In summary, the results are summarized as: (i) ferromagnetic $J_{\|}^{n n}$ increases with the Mn-O-Mn angle $\phi$, whereas antiferromagnetic $J_{\|}^{n n}$ is rather constant with $\phi$; (ii) $P_{e_{g}}$ increases with $\phi$ and decreases with $l$, whereas $P_{t_{2 g}}$ decreases with $s$ and partially cancels $P_{e_{g}}$; and (iii) orbital ordering, a needed ingredient in the rising of $P$, is however saturated along the series and does not influence the trend of polarization as a function of $R$.

Therefore, the main message is that one can expect high ferroelectric polarization with large $\phi$ and small $l$ in the AFM-E phase; however, such large $\phi$ would simultaneously increase the ferromagnetic $J_{\|}^{n n}$, therefore stabilizing the centrosymmetric AFM- $A$ spin configuration instead of the polar AFM- $E$ phase. The problem is then how to increase $P$, still keeping the AFM- $E$ phase as stable spin-state (i.e., via strain, pressure, alloying, etc.): we hope that our findings will be helpful to answer this question. 


\section{ACKNOWLEDGMENTS}

We thank Elbio Dagotto and Claude Ederer for their careful reading of the manuscript. The research leading to these results has received funding from the European Community's Seventh Framework Programme (FP7/2007-2013) under grant agreement No. 203523-BISMUTH. K.Y. acknowl- edges support from bilateral agreement between Consiglio Nazionale delle Ricerche and Deutsche Forschungsgemeinschaft (DFG). Computational support from Barcelona Supercomputing Center and Cineca Supercomputing Center is gratefully acknowledged. In this paper, the crystal structure and WFs are plotted using the program XCRYSDEN. ${ }^{37}$ *silvia.picozzi@aquila.infn.it

${ }^{1}$ S. W. Cheong and M. Mostovoy, Nat. Mater. 6, 13 (2007).

${ }^{2}$ A. P. Levanyuk and D. G. Sannikov, Sov. Phys. Usp. 17, 199 (1974).

${ }^{3}$ M. Iizumi, J. D. Axe, G. Shirane and K. Shimaoka, Phys. Rev. B 15, 4392 (1977).

${ }^{4}$ T. Kimura, T. Goto, H. Shintani, K. Ishizaka, T. Arima, and Y. Tokura, Nature (London) 426, 55 (2003).

${ }^{5}$ I. A. Sergienko, C. Şen, and E. Dagotto, Phys. Rev. Lett. 97, 227204 (2006).

${ }^{6}$ M. Mostovoy, Phys. Rev. Lett. 96, 067601 (2006).

${ }^{7}$ I. A. Sergienko and E. Dagotto, Phys. Rev. B 73, 094434 (2006).

${ }^{8}$ H. Katsura, N. Nagaosa, and A. V. Balatsky, Phys. Rev. Lett. 95, 057205 (2005).

${ }^{9}$ S. Picozzi, K. Yamauchi, B. Sanyal, I. A. Sergienko, and E. Dagotto, Phys. Rev. Lett. 99, 227201 (2007).

${ }^{10}$ B. Lorenz, Y. Q. Wang, and C. W. Chu, Phys. Rev. B 76, 104405 (2007); B. Lorenz, Y. Q. Wang, Y. Y. Sun, and C. W. Chu, ibid. 70, 212412 (2004).

${ }^{11}$ J.-S. Zhou, J. B. Goodenough, J. M. Gallardo-Amores, E. Morán, M. A. Alario-Franco, and R. Caudillo, Phys. Rev. B 74, 014422 (2006).

${ }^{12}$ M. Tachibana, T. Shimoyama, H. Kawaji, T. Atake, and E. Takayama-Muromachi, Phys. Rev. B 75, 144425 (2007).

${ }^{13}$ T. Mori, N. Kamegashira, K. Aoki, T. Shishido, and T. Fukuda, Mater. Lett. 54, 238 (2002).

${ }^{14}$ J. A. Alonso, M. J. Martínez-Lope, M. T. Casais, and M. T. Fernández-Díaz, Inorg. Chem. 39, 917 (2000).

${ }^{15}$ E. O. Wollan and W. C. Koehler, Phys. Rev. 100, 545 (1955).

${ }^{16}$ J. P. Perdew, K. Burke, and M. Ernzerhof, Phys. Rev. Lett. 77, 3865 (1996).

${ }^{17}$ G. Kresse and J. Furthmüller, Phys. Rev. B 54, 11169 (1996).

${ }^{18}$ N. Marzari and D. Vanderbilt, Phys. Rev. B 56, 12847 (1997); I. Souza, N. Marzari and D. Vanderbilt, ibid. 65, 035109 (2001).

${ }^{19}$ M. Posternak, A. Baldereschi, S. Massidda, and N. Marzari, Phys. Rev. B 65, 184422 (2002).

${ }^{20} \mathrm{http}: / / \mathrm{www} . f l a p w . d e$

${ }^{21}$ E. Wimmer, H. Krakauer, M. Weinert, and A. J. Freeman, Phys.
Rev. B 24, 864 (1981).

${ }^{22}$ F. Freimuth, Y. Mokrousov, D. Wortmann, S. Heinze, and S. Blügel (unpublished).

${ }^{23}$ L. Nordström and A. Mavromaras, Europhys. Lett. 49, 775 (2000); M. Divis, K. Schwarz, P. Blaha, G. Hilscher, H. Michor, and S. Khmelevskyi, Phys. Rev. B 62, 6774 (2000).

${ }^{24}$ We recall that the polarization is obtained as a vectorial sum of the displacement of the Wannier-function centers with respect to the ionic positions + the contributions due to the point-charge model.

${ }^{25}$ R. D. King-Smith and D. Vanderbilt, Phys. Rev. B 47, 1651 (1993).

${ }^{26}$ R. Resta, Rev. Mod. Phys. 66, 899 (1994).

${ }^{27}$ In our previous calculations (Ref. 9), our reference paraelectric structure was different from AFM- $A$ chosen here and showed some noncollinear spin arrangements. However, the different path does not affect the final results as far as the polarization is concerned.

${ }^{28} \mathrm{Ph}$. Ghosez, J.-P. Michenaud, and X. Gonze, Phys. Rev. B 58, 6224 (1998).

${ }^{29}$ T. Kimura, S. Ishihara, H. Shintani, T. Arima, K. T. Takahashi, K. Ishizaka, and Y. Tokura, Phys. Rev. B 68, 060403(R) (2003).

${ }^{30}$ J. B. Goodenough, Phys. Rev. 100, 564 (1955); J. Kanamori, J. Phys. Chem. Solids 10, 87 (1959).

${ }^{31}$ W. G. Yin, D. Volja, and W. Ku, Phys. Rev. Lett. 96, 116405 (2006).

${ }^{32}$ V. I. Anisimov, F. Aryasetiawan, and A. I. Lichtenstein, J. Phys.: Condens. Matter 9, 767 (1997).

${ }^{33}$ S. Picozzi, K. Yamauchi, G. Bihlmayer, and S. Blugel, Phys. Rev. B 74, 094402 (2006).

${ }^{34}$ A. Munoz, M. T. Casais, J. A. Alonso, M. J. Martinez-Lope, J. L. Martinez, and M. T. Fernandez-Diaz, Inorg. Chem. 40, 1020 (2001).

${ }^{35}$ S. Okamoto, S. Ishihara, and S. Maekawa, Phys. Rev. B 65, 144403 (2002).

${ }^{36}$ A. Filippetti and N. A. Hill, Phys. Rev. B 65, 195120 (2002).

${ }^{37}$ A. Kokalj, Comput. Mater. Sci. 28, 155 (2003); http:// www.xcrysden.org/ 